The treatment should be aimed at increasing the oxidizing power of the system. If the disease is associated with anæmia and chlorosis the iron preparations are indicated. Prout recommended the long-continued use of nitromuriatic acid, which our patient considered was of much benefit to him. Bartels, however, found it of no value after two months of steady use. He considers that, since the only danger lies in the tendency to form concretions, and since we cannot prevent its formation, the indication is to furnish solvents for it by giving diluent drinks ; and in his case, in which, however, the amount of urine passed was habitually small (one thousand and seventy-eight cubic centimeters), the patient was free from danger when the arnount of urine was increased by Seltzer water to fourteen hundred or fifteen hundred cubic centimeters.

Alkalies, although dissolving cystin, should not be used, since the cystin is sometimes deposited in alkaline urine, and since the earthy phosphates, which are sure to be separated by alkalies, may be deposited upon and increase the size of a cystin concretion already existing.

\title{
THE PSYCHOLOGICAL AND PATHOLOGICAL NATURE OF GENERAL PARALYSIS AND ITS TREATMENT.
}

BY EDWARD C. MANN, M. D.,

Superintendent Sunnyside Medical Retreat for Mental and Nervous Diseases, Washington Heights, New York; Late Medical Superintendent New York State Em. Insane Asylum.

Psychology. - It is doubtless true that in certain states of the brain, mental action or the actions of the higher centres of the brain may at times become automatic, and may be performed without even the intervention of consciousness. A constant repetition of any given mental action causes it to become organically registered in the brain centres; so that while at first a series of thoughts are performed consciously by the individual, they ultimately become reflex, responding to the recognized stimulus without consciousness, and independent of any effort or intervention of this condition. It is owing to injury of this mind power that we must look for an explanation of the mental symptoms of general paralysis.

Two classes of mental actions will necessarily be involved in this disease : first, those which are of so recent an origin as not to have become organically registered; and second, those which are still unable to be performed without conscious interference. One of the most powerful instincts or ideas in the human mind is the importance of self. In a healthy state we draw up and surround ourselves with an ideal self, which, if we are healthy minded, finds no expression. If the power to which all mental processes are due be impaired, those actions, amongat 
others, will be affected which are the most detailed and elaborate, the most varied, the least rigidly defined, and the least organically registered. Of all mental processes, those involved in the consideration of self are at once the most general, extensive, changeable, and complicated, as well as the most vague and undefined. Self occupies in the mind the widest, most frequent, and most capricious attention. As in general paralysis this mind power is the seat of the main lesion, the psychical processes concerned in the consideration of self will be the first implicated, and will present the most prominent symptoms. In general paralysis, the ideal self runs riot, - the man, not as he is, but as he has pictured himself, and as he would have himself be. In the inception of his disease the patient feels himself " bang up" and "perfect;" everything is "elegant" and rose-colored. His wealth is unbounded, and he orders " a million" cigars and palaces built of gold and diamonds with the utmost indifference and nonchalance, thoroughly believing in his capacity to do all these things. The patient's delusions are markedly progressive in number, absurdity, and exaggeration. Being rather feeble, he imagines himself capable of immense sustained exertions. In general paralysis, the mental processes which are the most automatic are the last to be affected; the patient entertains perfectly reasonable ideas about his actual self, and, although possessing thousands of ideal dollars and estates, will tell you correctly that he earns but ten dollars per week, as this idea has, from frequent and constant repetition, become automatic. In the same manner, a patient under my care tells me correctly that his suit of clothes cost fifteen dollars in London, and in the same breath says that he has ordered a silk velvet suit with diamond buttons. At the present moment he considers his health perfect, but acknowledges that in the past he has had many sicknesses and infirmities. Matters of recent occurrence which have not had opportunities for repetition, and so have not become organically registered and automatic, and which therefore involve consciousness, are far more dependent upon mind than matters of earlier date which have been so frequently repeated as to acquire automaticity. For this reason, patients suffering from general paralysis, while stating with accuracy events and detailed accounts of the past, can give but a very vague and confused history of the events of the past few days or weeks. This defect in memory is consequently one of the most noticeable symptoms in the early stage of the disease, and we notice it particularly in persons of methodical habits. Leaving this interesting field of the psychology of general paralysis, we proceed to notice its pathology.

Pathology. - The pathology of general paralysis is very obscure, and invites especial attention at the hands of the profession. The fundamental lesion of this disease is, in the writer's opinion, a general diffused interstitial encephalitis which involves accessory structural changes of 
different character. The posterior columns of the cord are also not unfrequently affected. The primary and most palpable form of the interstitial degeneration is colloid, where the transformed matter is presented under the aspect of a hyaline substance, semi-transparent, slightly refractory, and at certain points of a bluish tint. When existing in isolated masses of small size, it preserves the form and aspect of whatever cerebral elements it may have invaded. This product of inflammation does not appear to be at all of a tubercular nature, neither is it fatty, being insoluble in ether or chloroform. It is not amyloid, because it is unaffected by tincture of iodine, solutions of potass and soda, and is dissolved in strong acetic acid. It is not organic, as there is no reaction with hydrochloric acid. Its solubility in hot water, especially when potass or soda is added, would seem to indicate a peculiar chemical composition. When we examine the cerebrum as the principal seat of paresis, we find the ependyma of the ventricles to be the centre, or perhaps one of many centres, of that destructive process which is indicated by the symptoms of general paralysis, which affects all parts of the encephalon, and produces those secondary pathological appearances which have previously been identified as the cause of the disorders of motility and sensibility which follow. The progress of the morbid degeneration from the point where the ventricles have become dilated, their ependyma thickened, where their surface, especially in the fourth ventricle, is covered with granulations, is probably upwards along the connective, involving all tissues as well, and is gradual and insidious, and can be traced only by means of the more advanced alterations in structure. This interstitial irritation, however disseminated, is propagated by nuclear proliferation, and invades the white matter in common with the cortical substance, and also the capillaries, which are thickened, tortuous, and massed together. The cells of the cortical portion are sometimes found infiltraterl with granulations, but retaining their form. This is found in the third stage of the disease. It is in the middle and inferior portions of the gray matter that the cells are observed to have brilliant nuclei tending towards colloid, while their normal aspect is preserved. The walls of the cell nearest the lesion are transformed into a shining, refractory hyaline substance, the colloid infiltration having been propagated to both. The microscopic as well as the naked-eye appearances, may arise first in the brain, and subsequently in the medulla and cord, or they may appear first in the cord and medulla, and afterwards in the brain; and they may also show themselves simultaneously. If the brain be primarily attacked, the psychical signs predominate, or are exclusively manifested. If the medulla be the primary seat of the disease, muscular pain, tremor, and ataxic symptoms, spreading gradually to the lips and tongue, and disturbance of the internal viscera corresponding to the portion of the spinal column 
involved, precede alienation and increase the difficulty of diagnosis. Finally, when the whole cerebro-spinal axis participates at once in the colloid degeneration, the characteristic indications of paresis will appear simultaneously or in rapid succession. It is important for us to bear in mind that the colloid degeneration is sometimes absent, and that we occasionally meet with it in brain disease which is only remotely connected with paresis.

I will now cite as concisely as possible the opinions of the highest authorities relative to the pathology of the disease under question.

Bayle designated the disease chronic arachnitis. Calweil considered it a diffuse chronic meningeal encephalitis, and held that it was of inflammatory origin. Baillarger observed two sets of anatomical alterations : congestion of the membranes and chronic hydrocephalus, with atrophy and softening of the brain. Brunet and Lancereaux speak of a neo-membrane or a pachymeningitis, the formation of which they explain by the exudation of a parietal layer from its walls, which is insensibly organiz $d$, and which bears the marks of fatty degeneration. The rupture of these vessels leads to the occurrence of arachnoid cysts. Erlenmaye explains the atrophy, which has been noticed, by the successive and repeated effusions of serum, the nervous elements being replaced by an amorphous substance. Frerichs considers the induration of the gray matter as connecting the pathological condition with sclerosis. The alterations in the white matter consist of hardening or softening, and increase of the fluid of the ventricles. M. Luys, who, by the way, is a very able investigator, believes that in the softening of the cortical substance of the cerebellum may be discovered the source of general paralysis, but Magnan asserts that this conviction of M. Luys is founded on exceptional cases. M. Luys gives as the result of his microscopic investigations the following results, namely, the vessels of the pia mater are of considerable size, the walls of the capillaries are incrustated with granulations, and the surface of the pia mater presents scattered cell granules and molecules and extravasated blood globules. The gray substance is greatly developed, with palpable vascular arborizations, often in the form of a plexus. The calibre of the capillariesis, almost always contracted by the incrustations of minute granules, or by cells deposited chiefly at the bifurcations. They are of irregular shapes, and often break down and discharge their contents. The nerve tubes are malformed, their contents escape, and they present little more than a mass of débris. Rokitansky has detected three distinct pathological appearances: first, a mass of connective tissue which embraces in its net-work the nervous element, and in chronic cases is stiff, fibrous, and induces adhesions of the pia mater; second, the varicose and broken condition of the tubes, while the cells appear swollen; third, the presence of amyloid or colloid bodies. Wedl's observations on these points are as fol- 
lows : that contraction of the capillaries and small blood-vessels of the cells upon their walls leads to obliteration, their conversion into bands of connective tissue, and the consequent impairment of the nutrition of the part. Dr. Ertzbischoff attributes the hyperæmia of the cortical layers to the extreme development of the embryoplastic element in the capillaries, which, by compressing the vessel without and within, diminishes and ultimately destroys the cavity. This stasis necessitates granulation and adhesion. The majority of German pathologists localize the disease in changes of the walls of the vessels, and in the development of the connective tissue. Meschede sees the essential characteristics of paresis in the degeneration of cerebral cells, especially those of the cortical substance, which he depicts as of abnormal shape, and filled and surrounded with fatty and pigmentary granulations. Lockhart Clarke speaks of the conversion of the cells of the convolutions into pigmentary bodies, irregularly shaped and about to break up. Contemporaneously with this change, the spinal marrow, especially in chronic cases, is softened to the consistency of cream, or there ma be a granular degeneration in the gray matter of the cord. In some cases Westphal has discovered granulation in the posterior column of the cord which did not extend higher than the peduncles of the cerebrum, so that the alteration could not be regarded as secondary or proceeding from the pathological condition of the convolutions.

The course of paresis generalis may very properly be divided into four stages: first, that of delirium, with or without slight physical lesions. Second, that of defective coördination of movement, exaggerated sentiments, alterations in the secretions, with continued delirium. Third, that of special dementia, with greater stupidity and degradation than in other forms, control of muscles diminished, habits dirty. Fourth, perception of impressions by external senses abolished, insensibility to pain, extinction of functions of relation and organic life, disturbance in circulation, complete adynamic ataxy, and marasmus. Optimism and ambitious ideas, as I remarked in the first part of this paper, constitute the essential mental characteristics. Previous to the establishment of thorough delirium or delusions there may be traced deviations from healthy mentalization, which, though faint or latent, should be accepted as prodromata. There is probably no doubt that the gradual evolution of physical and psychical symptoms corresponds intimately with structural alterations in the nervous centres. The employment of galvanization of the brain and cervical sympathetic with good results in some cases of general paralysis, has suggested to the author the following question: Whether some of the principal changes occurring in paresis may not have their origin in a congestion originating in the ganglia of the sympathetic, transmitted along the spinal cord, ultimately involving every tissue within the cranium, and eventuating simultaneously in the degeneration of blood vessels, cells, and nerve tubes, and the men- 
tal and motor perversions which in 80 marked a manner distinguish general paralysis from all other diseases of the same class.

The diagnostic symptoms of general paralysis, aside from the exalted notions, are difficulty in articulation, with a trembling of the tongue when it is protruded beyond the teeth, often a turning of it to one side, and a general inability to use it freely, as if it were too large for the mouth, or too heavy for use, very flabby, and easily indented by the teeth; a peculiar dragging of one of the feet or legs; added to these symptoms will be found a heavy, dull expression of countenance, an unusual appearance of the eyes, and almost invariably an unequal contraction of the pupils. It requires close observation to detect these symptoms in the early stages, and careful treatment may relieve them temporarily; but although we may retard the issue of the disease by watchful care and skillful treatment, it inevitably advances insidiously to paralysis of the tongue and limbs, and enfeeblement of the mind. In the last stage, which sometimes lasts for years, emaciation succeeds obesity. Sometimes there is intense restlessness, but generally we find lethargy of body as well as of mind, this lethargy being disturbed by twitchings or epileptiform convulsions. These often terminate life, but the most frequent causes of death are sheer exhaustion and tubercular disease. With regard to the condition of the retina in general paralysis, we find the nerve changes generally proportionate to the contraction and dilatation of the pupils : the contraction of the pupils corresponding to the early or hyperæmic stage, and the dilatation of the pupils to the white atrophic condition of the optic disc. With regard to the temperature, $I$ have noticed as an unvarying symptom that there is always a higher temperature in the evening than in the morning, seldom less than the difference of one degree, and by the thermometer we may discover the progress of the disease when we cannot do it satisfactorily by any other means. In sleepless and destructive patients the temperature is higher than in quiet cases. We may consider the average duration of general paralysis to be about thirteen months, very few patients living more than three years after the development of well-marked symptoms.

\section{THE EFFECTS OF AN OVERDOSE OF GELSEMINUM SEM- PERVIRENS. ${ }^{1}$}

BY F. W. Goss, M. D.

SePtember 10, 1878, at eleven A. M., a lady of nervous temperament and subject to neuralgic headache, mistaking the dose, swallowed a teaspoonful of Metcalf's fluid extract of gelseminum. One hour later she became alarmed on account of dimness of vision, drooping of the

1 Read before the Boston Society for Medical Improvement, October 14, 1878. 